\title{
Combining Virtual and On-site Teaching for Innovation Training in Robot Engineering
}

\author{
Xudong Pan ${ }^{1}$, Hong Huo ${ }^{2}$, Yu Jiang ${ }^{3}$, Xinyu Cui ${ }^{4}$, \\ Bo Peng ${ }^{5}$, Haibo $\mathrm{Gao}^{6}$, Xiuqin $\mathrm{Han}^{7}$
}

\begin{abstract}
${ }^{1}$ Engineering Innovation Practice Center, School of Mechatronics Engineering, Harbin Institute of Technology, No.92, Xidazhi Street, Nangang District, Harbin, China (pxd@hit.edu.cn) ORCID 0000-0002-8574-7167; '2Engineering Innovation Practice Center, School of Mechatronics Engineering, Harbin Institute of Technology, No.92, Xidazhi Street, Nangang District, Harbin, China (huohong@hit.edu.cn) ORCID 0000-0001-9092-0713; ${ }^{3}$ Engineering Innovation Practice Center, School of Mechatronics Engineering, Harbin Institute of Technology, No.92, Xidazhi Street, Nangang District, Harbin, China (460993735@qq.cn) ORCID 0000-0001-5282-4210; ${ }^{4}$ Mechanical Engineering, School of Mechatronics Engineering, Harbin Institute of Technology, No.92, Xidazhi Street, Nangang District, Harbin, China (826523168@qq.cn) ORCID 0000-00019426-774X; ${ }^{5}$ Engineering Innovation Practice Center, School of Mechatronics Engineering, Harbin Institute of Technology, No.92, Xidazhi Street, Nangang District, Harbin, China (305663761@qq.cn) ORCID 0000-0001-9147-1657; 6Department of Aeronautics and Astronautics Manufacturing Engineering, School of Mechatronics Engineering, Harbin Institute of Technology, No.92, Xidazhi Street, Nangang District, Harbin, China (gaohaibo@hit.edu.cn) ORCID 0000-0002-6501-656X; 7Engineering Innovation Practice Center, School of Mechatronics Engineering, Harbin Institute of Technology, No.92, Xidazhi Street, Nangang District, Harbin, China (hangxq@hit.edu.cn) ORCID 0000-0003-1906-8163
\end{abstract}

\begin{abstract}
Innovation training in engineering is an important facet of technical education in universities across China. Robotics training is a new course that has been developed using an informatization-based engineering training system at the Engineering Innovation Practice Center of the Harbin Institute of Technology. In this study, we propose a pedagogical method that combines virtual and on-site modes of teaching. Feedback obtained from students enrolled in the course showed a positive response to it. They claimed that they gained valuable knowledge from it that enabled them to carry out the relevant tasks in a better manner and more quickly. The feasibility of the proposed scheme was verified by a virtual simulation.
\end{abstract}

Author Keywords. Innovation Training in Engineering, Virtual and On-site Learning, Robotics.

Type: Research Article

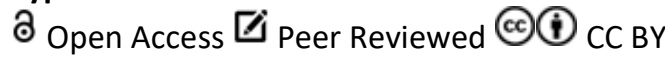

\section{Introduction}

With the emergence and rapid development of new technologies, the next two decades are expected to usher in a transformation from traditional industries to new industries (Ke et al. 2020). Robotics training is a recently developed course that is designed to closely track such developments in the context of the design and use of robots for various tasks. It can help cultivate practical skills and comprehensive engineering capability among students. To improve the efficiency of learning, it uses a method of pedagogy that combines virtual and onsite teaching. Students first learn the structure of the robot through virtual simulations, learn to virtually assemble it, and then engage in its physical design.

Virtual assembly refers to a type of assembly in a virtual engineering environment that uses model data and visualization (Peng 2017). It is used to quickly assemble parts into a product 
in a virtual engineering environment and represents a method of product assembly aided by computers (Meng 2014). The key to virtual assembly technology is to discover the relations between parts and form a plan of the sequence of assembly of products (Anchao 2018).

Since the 1990s, a growing number of researchers have studied the application of virtual assembly to a variety of industries. The VRCM Laboratory in Washington State University has worked with the National Institute of Standards and Technology (NIST) in the United States to create a virtual assembly design environment (Jayaram et al. 1999). The Fraunhofer Institute of Industrial Engineering in Germany has also developed an interactive system using virtual human models to formulate plans for virtual assembly (Blach et al. 1998). The Department of Chemical and Mechanical Engineering of Heriot-Watt University in the UK has developed a channel design and assembly system (CHDP) based on Virtual Reality (VR) technology. The CG National Key Laboratory of Zhejiang University in China has developed an integrated virtual design and virtual assembly system based on several channels (Wan, Gao, and Peng 2002). The Digital Design and Manufacturing Laboratory of Beijing University of Technology in China has developed an integrated virtual assembly process planning system (Liu, Ning, and Yan 2006), and the Experiment Center of Guangzhou University has developed an experimental system for virtual assembly for disabled people (Hongjian and Zhe 2013). Yi Chen published an article entitled real-time 3D unstructured environment reconstruction utilizing VR and Kinect-based immersive teleoperation for agricultural field robots (Chen et al. 2020).

A combination of virtual and on-site training methods is used in innovation training in the context of robot engineering. In view of the time-consuming disassembly and assembly of robotic components, as well as the difficulty of systematization and automation in the operational process, a robot component assembly system based on virtual simulation technology has been developed to cooperate with practical teaching. The system combines virtual simulation technology with equipment used for an immersive experience, thus, allowing students to assemble robotic components through the interactive equipment as they would in the physical environment. This improves their immersive and interactive experience of the assembly process. In addition, the system displays information on the parts, examples of assembly, guidance for the assembly process, and monitoring functions. Once their virtual assembly has passed an assessment, the students form teams to independently select robotic components, sensors, and motors according to the requirements of the project at hand, and assemble them to design robots for different tasks. These teams then compete with one another to determine the best design and implementation.

This method of teaching can arouse the students' interest in robots and allow them to understand the working principles of robots. The problem of the time-consuming disassembly and assembly of the robotic components is also solved by it. It shortens the time that students need to spend to design and optimize the structure of robot. It has led to personalized student-centered training, while striking a balance between knowledge and ability, and research and practice. By the benefits of this method of teaching, it also plays an important role during the COVID-19 pandemic. Virtual simulations, and modes of online and offline teacher-student interactions were used owing to safety-related concerns.

\section{Virtual Teaching}

According to the concept of pedagogy that combines virtual and empirical environments, we develop here a software for the virtual assembly of robotic parts based on virtual simulation technology. The software consists of four system functions: the introduction of information on the parts, animations of examples of assembly, virtual assembly, and assigning scores to 
assembly training, as shown in Figure 1. The information on robotic parts and examples of assembly are provided before the students are asked to perform the virtual assembly task, which can help them better understand common parts and master the structure of robotic transmission. The students can check their assembly scores after completing the virtual training.

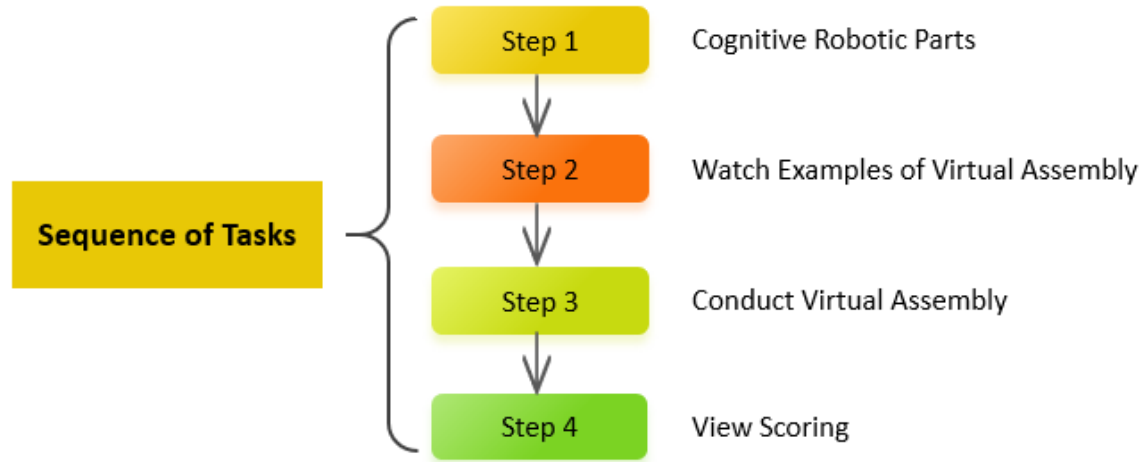

Figure 1: Sequence of virtual assembly tasks

\subsection{Introducing Information on Robotic Parts}

Once the function for introducing the students to information on parts of the robot is turned on, the initial interface displays small icons of all parts as shown in Figure 2. When an icon is clicked, the information on and specifications of the relevant part pop up as shown in Figure 3.

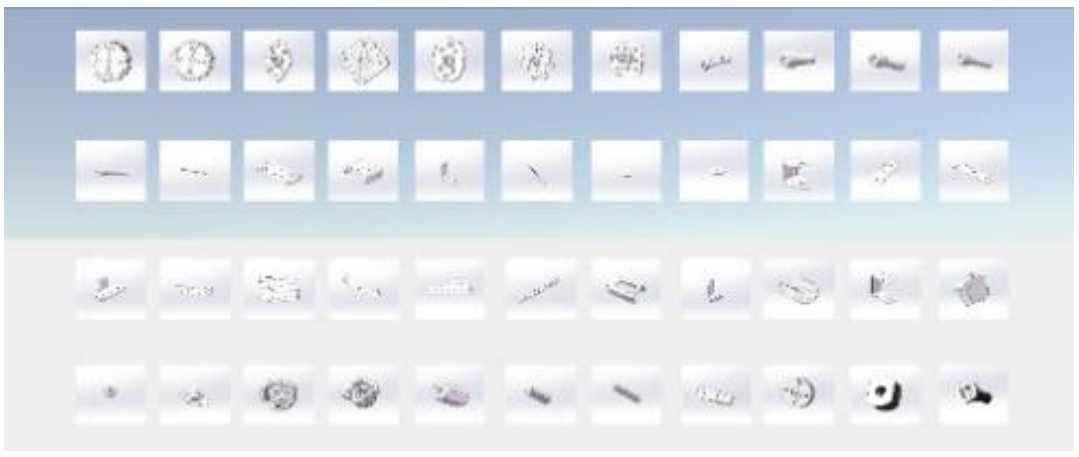

Figure 2: Interface for introducing parts' information

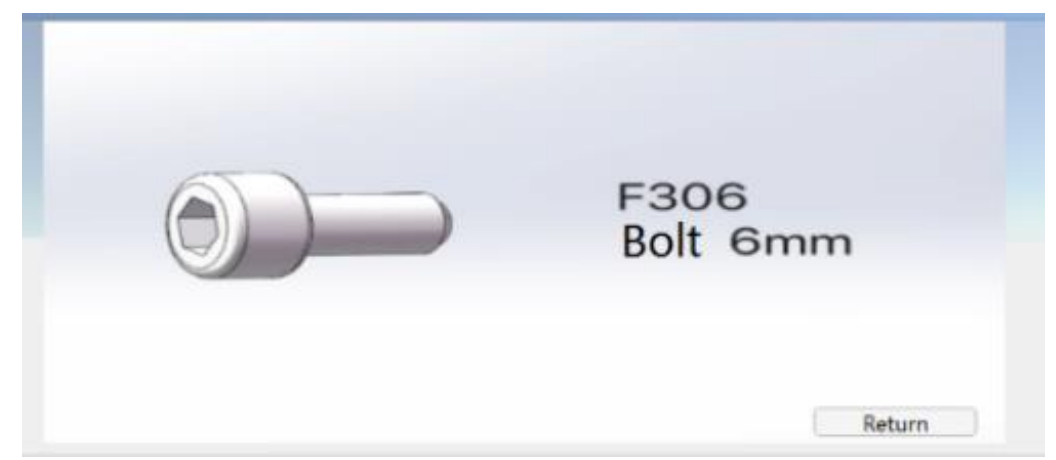

Figure 3: Another view of the interface for introducing parts' information

\subsection{Examples of virtual assembly}

In this session, schematic processes for the assembly of two robotic cars are provided as animations as shown in Figure 4. The animation for the assembly of robotic car 1 is divided into three parts: assembly of the wheel, claw, and the overall structure. That for the assembly of robotic car 2 is similarly divided into the assembly of the wheel, body, and the overall 
structure. When a student clicks the "Assembly Example" button, the relevant animation is played. By watching it, the student can understand the process of robot assembly and the corresponding procedures.
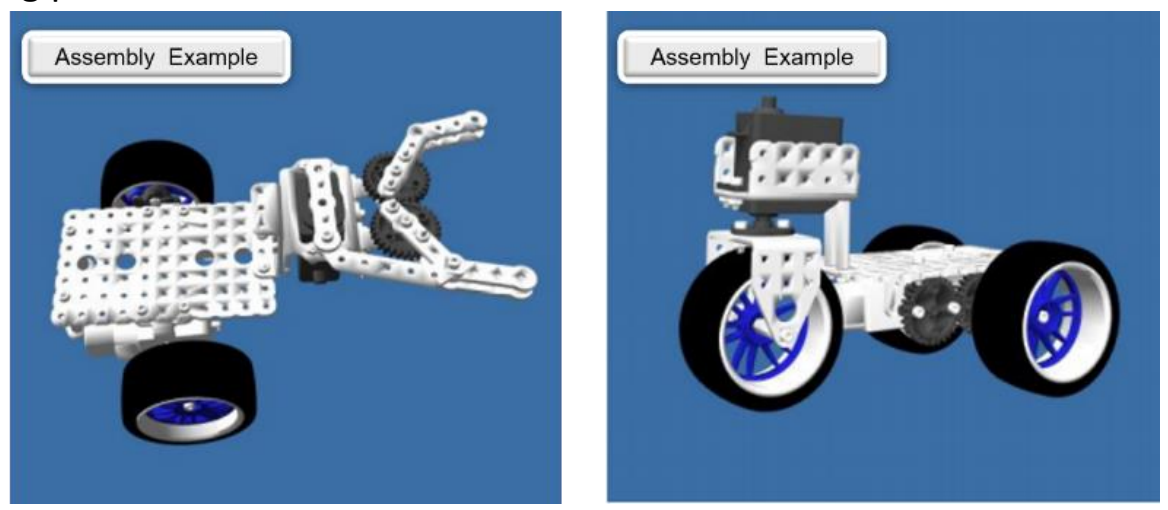

Figure 4: Assembly example animation of robotic cars

\subsection{Virtual assembly}

To enable students to better grasp the methods of assembling robots to improve the efficiency, quality, and effect of such an assembly in practice, we refine the process in the virtual training for the assembly. The procedure for the assembly of robotic car 1 includes training on assembling its head, steering gear, hands, torso, legs, and its overall structure. That for the assembly of robotic 2 includes training on assembling its mechanical claws, arms, torso, and the overall structure. All assembly processes have collision detection functions. When parts of the robot used by the student collide with other parts, it cannot be assembled, and the path of assembly needs to be changed. The initial assembly interface is shown in Figure 5, when "Start" is pressed, students can perform virtual assembly. Figure 6 shows the assembly process.

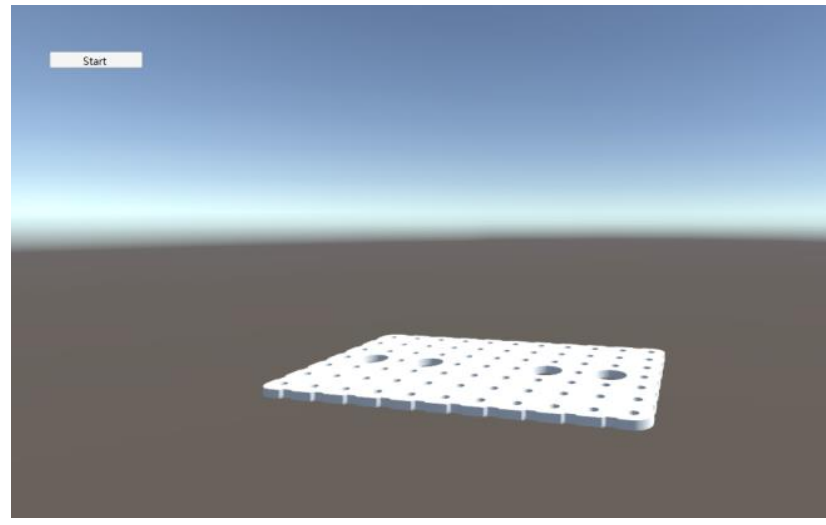

Figure 5: Initial interface

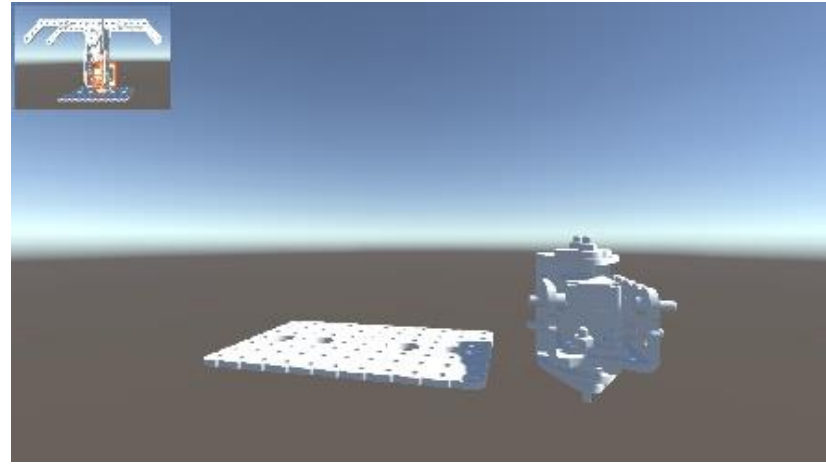

Figure 6: Assembly process 
The entire process of virtual assembly can be performed on a PC or through hardware for Virtual Reality. This kind of training enables the student to see the three-dimensional (3D) and immersive effects of the parts on the overall design.

\subsection{Scoring}

Once students have completed training for robot assembly, they can view their scores on it by clicking the "submit" button (Figure 7). The initial score is zero. When a part to be assembled is dragged to a specified position, the corresponding score is added. The score depends on the complexity of the parts to be assembled, and the score for each step is 100 divided by the total number of steps. The student's assembly score is the sum of his/her corresponding scores on all parts. The highest attainable score on assembly training is 100 .

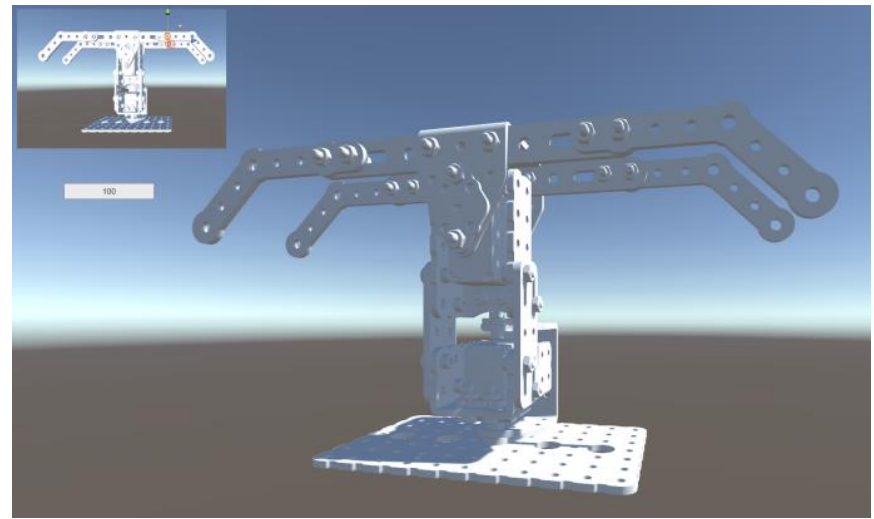

Figure 7: Scoring interface

The software used for robot assembly based on virtual simulation technology is simple and easy to operate. There is no need to install new applications. The user simply needs to open the compiled, independent .exe file to enter the execution interface. The training system is also easy to modify, and new parts and functions can be added to it at any time by executing them as a new, exe file.

\section{On-site Teaching}

On-site teaching is carried out after the completion of the virtual teaching process. Targeted practical projects are set-up according to the specialization of students attending the course, such as inspection robots, dancing robots, handling robots, and fighting robots. We use the "cliff robot" as an example to illustrate the contents and sessions of practical teaching.

A "cliff robot" has a self-aware function whereby it can autonomously determine whether the front is a "cliff edge". When the walking robot meets a "cliff edge", it stops to move forward. When being taught on site, the students are required to analyze the relevant requirements, design and install the mechanical structure, program the robot, and test and debug the entire machine. The design and installation of the mechanical structure consists of five parts: installing the driving wheel, driven wheel, wheel bracket, track, and electronic components, as shown in Figure 8.

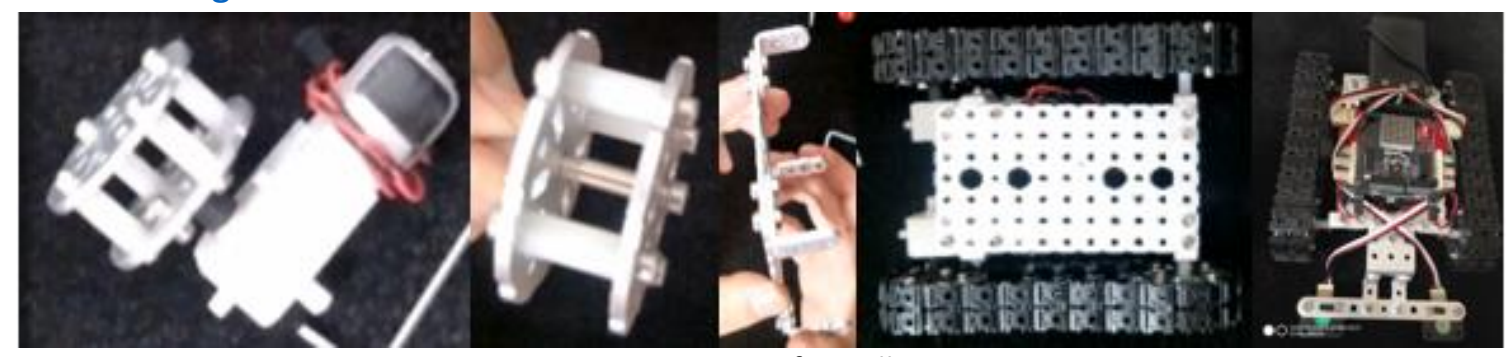

Figure 5: Steps of installation 
Some notes on the installation procedure are as follows:

- Before installing the driving wheel, a self-tapping screw must be inserted in advance because it cannot be subsequently inserted.

- A shaft sleeve must be added between the center hole of the driven wheel and the screw to act as a bearing.

- The motor bracket and the chassis of the robotic training are connected as a rigid structure, because of which at least two screws are required.

- The track is composed of multiple pieces that can be set to any length.

- When installing an infrared sensor, the student should be careful not to cover the transmitter and receiving heads to avoid deviations in the results of detection.

\section{Teaching During the Pandemic}

During the COVID-19 pandemic, virtual simulations, and modes of online and offline teacherstudent interactions were used owing to safety-related concerns.

Teachers used modular fighting robots as the main content of the course. The students learned the structure of the robot through the virtual simulation software. After acquiring the requisite theoretical knowledge of modules for robot control and programming online, they designed the fighting robots by themselves. After completing online programming based on Arduino, the students sent their design ideas and corresponding programs to the teacher through a teaching platform. The teacher built the robot according to this design and installed the relevant program into it. The teacher then used a live broadcast to show the entire process of robot confrontation to the students, which allowed them understand that only a reasonable mechanical structural design and logically rigorous program would lead to successful implementation. This minimizes the potential shortcomings of the use of online teaching for practice-oriented courses.

\section{Course Evaluation Rubric}

After one-year of teaching them by combining the virtual and the empirical environments, the feedback obtained from the students was as follows:

- Assembling robots through a virtual simulation is easy to understand and is conducive to clearly displaying the procedures involved. The sequence of installation of the robotic structure is important. At the beginning of the class, the students did not know where to start. Through the virtual simulation, however, they were able to clearly understand the sequence of installation and the consequences of various related decisions.

- Through the virtual assembly of robots, the students were able to understand the entire assembly process conveniently and quickly, which helped them implement the combination of the virtual and empirical environments as well as the suitable combination of theory and practice.

- This method of teaching method is more useful and attractive than traditional pedagogy. It allows the student to comprehend the power of technology and the virtual environment.

- The results of learning were examined through competition (PK) between groups of students. Several sets of robots were assembled by them using Virtual Reality technology, and the best solution was chosen as the winner of the competition. 


\section{Conclusions}

The combination of virtual and on-site teaching for robotics focuses on the students' ability to analyze and solve problems while helping them accomplish the objectives of the course and understand aspects of the enterprise that are difficult to comprehend in practice. Through a combination of virtual and on-site operation, an effective environment for learning can be created. In class, students can learn the composition of the robot through virtual simulations and undertake its virtual assembly. They can then design the structure of the robot and choose sensors according to the requirements of the project. Finally, they can be assessed through group competition. This helps complement their theoretical and practical knowledge. It is also a useful mode of teaching during the pandemic.

\section{References}

Anchao, H. 2018. "Virtual simulation of ship sectional assembly based on VR technology". Master's thesis, Jiangsu University of Science and Technology. https://kns.cnki.net/kcms/detail/detail.aspx?dbcode=CMFD\&dbname=CMFD201901\&file name $=1018848010$. nh\&v=ugu75\%25mmd2Fd51C756hCFKr9GMPEdWT6Qfi5bf5PI\%25m md2BZpXTXcM4PcUXI5FDy2kCMTE9ikv.

Blach, R., J. Landauer, A. Rösch, and A. Simon. 1998. "A highly flexible virtual reality system". Future Generation Computer Systems 14, no. 3: 167-78. https://doi.org/10.1016/S0167739x(98)00019-3.

Chen, Y., B. Zhang, J. Zhou, and K. Wang. 2020. "Real-time 3D unstructured environment reconstruction utilizing VR and Kinect-based immersive teleoperation for agricultural field robots". Computers and Electronics in Agriculture 175: 105579. https://doi.org/10.1016/j.compag.2020.105579.

Hongjian, L., and Q. Zhe. 2013. "Development of virtual assembly experiment system based on Kinect somatosensory interaction technology". Experimental Technology and Management 7: 98-102.

Jayaram, S., W. Yong, U. Jayaram, K. Lyons, and P. Hart. 1999. "A virtual assembly design environment". In Proceedings IEEE Virtual Reality, 172-79. https://doi.org/10.1109/VR.1999.756948.

Ke, Z., J. Rendong, K. Hongyan, Z. Zhiyi, and Z. Tian. 2020. "Construction of a practice system for the cultivation of outstanding engineering talents under the background of new engineering disciplines". Education Teaching Forum 7: 213-16.

Liu, J., R. Ning, and Y. Yan. 2006. "Research on integrated virtual assembly process planning system". Chinese Journal of Mechanical Engineering 23: 2486-91.

Meng, N. 2014. "Design and implementation of a virtual assembly training and evaluation system for automobile engines based on Unity3D". Master's thesis, Beijing University of Technology.

Peng, H. 2017. "Research and implementation of distributed collaborative virtual assembly simulation based on Unity3D". Master's thesis, Xiangtan University. https://kns.cnki.net/kcms/detail/detail.aspx?dbcode=CMFD\&dbname=CMFD201801\&file name=1017256909.nh\&v=PWo8dPta2dl3PD4992Bqc\%25mmd2Fs\%25mmd2FswfDxfW8s Nj7OaFdCWcBu6KfZNwVExorxnlY0S6n.

Wan, H., S. Gao, and Q. Peng. 2002. "VDVAS: An integrated virtual design and virtual assembly environment". Journal of Image and Graphics 7, no. 1: 27-35. 


\section{Acknowledgments}

The authors acknowledge the funding support from Heilongjiang Province 2020 Education and Teaching Reform Key Project of Higher Education (SJGZ20200047), Heilongjiang Province 2019 Education and Teaching Reform Project of Higher Education (SJGY20190180), Heilongjiang Province Education Science Planning "145" Planning Key Project (GJB1421036) and Harbin Institute of Technology COVID-19 Pandemic Reflections on Education Reform Project (XYZ2020008). 